\title{
Bilanz der Hartz-Reformen im Bereich der Arbeitsvermittlung
}

Die Neuausrichtung der Arbeitsvermittlung wurde vom Wissenschaftszentrum Berlin für Sozialforschung (WZB) gemeinsam mit infas Bonn für den Zeitraum 2004 bis Frühjahr 2006 im Rahmen der vom Bundesministerium für Arbeit und Soziales geförderten Evaluation der Hartz I-III-Gesetze untersucht. Dieser Beitrag fasst die Hauptergebnisse und Empfehlungen dieser Studie zusammen, die sich ausschließlich auf die Arbeitsvermittlung der Arbeitsagenturen und die Vermittlungsinstrumente im Geltungsbereich des SGB III beziehen. Die SGB-II-Reformen (Hartz IV) sind hingegen Gegenstand einer noch laufenden, separaten Evaluation des BMAS, die in diesem Beitrag außer Betracht bleiben.

\section{Hugh Mosley*}

\section{Das BA-Kundenzentrum}

Das Kundenzentrum, das neue Organisationsmodell der Arbeitsagenturen, ist eines der Kernstücke der Reform der Bundesagentur für Arbeit. Im Rahmen der Evaluierung wurden im Frühjahr 2005 und 2006 repräsentative Befragungen aller Agenturen unternommen und die Umsetzung der Reform in zehn Arbeitsagenturen genauer untersucht. ${ }^{1}$ Das Fazit der Studie lautet: Der Service für Arbeitslose und Arbeitgeber hat sich verbessert. Jedoch wird für Arbeitsuchende mit den größten Schwierigkeiten am Arbeitsmarkt (Betreuungskunden) zu wenig getan.

\section{Verbesserte Arbeitsabläufe}

Die Arbeitsabläufe im Kundenzentrum und der seit 2002 verbesserte Betreuungsschlüssel haben grundsätzlich die Rahmenbedingungen verbessert, um hohe Dienstleistungsqualität in Vermittlung und Beratung erreichen zu können (siehe Abb. 1). Zu nennen ist die Vorfiltrierung der Anliegen der Arbeitslosen und Arbeitsuchenden in einem
Abbildung 1: Ablauforganisation im Kundenzentrum

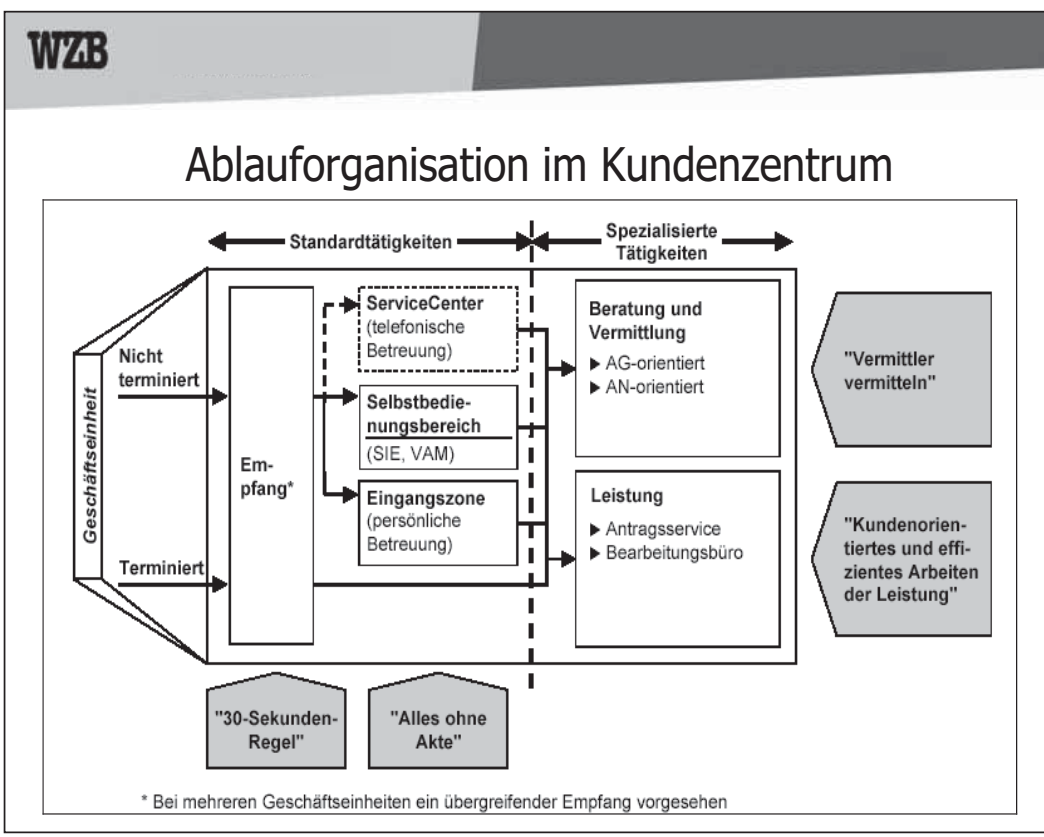

Quelle: BA 2004.
* Hugh Mosley, Wissenschaftszentrum Berlin für Sozialforschung.

Die Studie wurde seitens des WZB durch Silke Gülker, Petra Kaps, Andreas Mauer, Hugh Mosley (Projektleiter), Kai-Uwe Müller, Frank Oschmiansky und Holger Schütz erarbeitet. Von infas Bonn haben Doris Hess, Reiner Gilberg und Helmut Schröder maßgeblich mitgewirkt. Kurz- und Langfassungen der Studie "Neuausrichtung der Vermittlungsprozesse" stehen zur Verfügung unter: http://www.wzb.eu/ars/ab/
Eingangsbereich und im Servicecenter (Callcenter), wo ein beträchtlicher Anteil der Anliegen bereits dort abschließend bearbeitet wird. Festgelegte Gesprächstermine ermöglichen die ungestörte Beratung sowie die Vermeidung langer Wartezeiten. Die Vermittlungsgespräche folgen einer klaren Struktur. In der arbeitgeberorientierten Vermittlung wurden die Personalressourcen erhöht und Qualitätsstandards eingeführt. Aufgrund der positiven Entwicklungstendenzen wäre daher die Organisationsform des Kundenzentrums für den SGB-III-Bereich zu verstetigen und zu optimieren. 


\section{Standortbestimmung (Profiling) und Handlungsprogramme Arbeitnehmer}

Im Vermittlungsgespräch soll eine Klärung folgender Themen stattfinden:

- berufliche Kenntnisse und Qualifikationen

- Einstellung/Motivation

- Hemmnisse (Mobilität, gesundheitliche Einschränkungen)

- spezifische Arbeitsmarktbedingungen.

Diese „Standortbestimmung“ bildet die Grundlage für die Kundengruppenzuordnung als Marktkunde, Beratungskunde Aktivieren, Beratungskunde Fördern, Betreuungskunde durch den Vermittler. Die so genannten Handlungsprogramme setzen auf diese Kundengruppensystematik auf und steuern die „Produktvergabe“. Wie aus Abbildung 2 hervorgeht, wird nicht nur der Zugang zu Maßnahmen, sondern auch die Kontaktdichte vorgegeben. Nach der betriebswirtschaftlichen Logik der Bundesagentur für Arbeit werden die verfügbaren Ressourcen eher auf die mittlere Gruppe der Beratungskunden konzentriert.

Die Evaluationsergebnisse sprechen dafür, die Kundenzentren beizubehalten und weiterzuentwickeln. Allerdings muss die Beratungsqualität im Vermittlungsprozess gesteigert werden. Das durchschnittliche Qualifikationsniveau der Vermittler wäre dazu anzuheben; außerdem müssten beraterische Freiräume sichergestellt werden. Im aktuellen Stand sind noch erhebliche Leistungsunterschiede zwischen den Vermittlern festzustellen. Defizite liegen zu einem Teil in der Fachlichkeit (z. B. Berufskunde), zu einem anderen Teil bei den Beratungsfertigkeiten inklusive der „weichen“ kommunikativen Kompetenzen (Gesprächsführung etc.).

\section{Abbildung 2: Handlungsprogramme Arbeitnehmer}

\begin{tabular}{|c|c|c|}
\hline \multicolumn{3}{|c|}{ Handlungsprogramme Arbeitnehmer } \\
\hline $\begin{array}{l}\text { Handlungsprogramm und } \\
\text { zugeordnete Kundengruppe }\end{array}$ & Ziele & $\begin{array}{l}\text { Nächstes } \\
\text { Gespräch }\end{array}$ \\
\hline 1. Vermittlung (Marktkunden) & Die schnellstmögliche Vermittlung & $<3$ Monaten \\
\hline $\begin{array}{l}\text { 2. Perspektivenänderung } \\
\text { (Beratungskunden Aktivieren) }\end{array}$ & $\begin{array}{l}\text { Die Entwicklung von Engagement, } \\
\text { Motivation für eine schnellst- } \\
\text { mögliche Vermittlung }\end{array}$ & $<1$ Monat \\
\hline $\begin{array}{l}\text { 3. Abbau Beschäftigungs-hürden } \\
\text { (Beratungs-kunden Aktivieren } \\
\text { \& Fördern) }\end{array}$ & $\begin{array}{l}\text { Die frühzeitige Ermittlung und } \\
\text { Beseitigung von Vermittlungs- } \\
\text { hürden }\end{array}$ & $<2$ Monate \\
\hline $\begin{array}{l}\text { 4. Qualifizierung } \\
\text { (Beratungskunden Fördern) }\end{array}$ & $\begin{array}{l}\text { Die Anpassung von Fähigkeiten und } \\
\text { Qualifikationen }\end{array}$ & 2-3 Monate \\
\hline $\begin{array}{l}\text { 5. Erhalt Marktfähigkeit } \\
\text { (Betreuungskunden) }\end{array}$ & $\begin{array}{l}\text { Die Vermeidung von Passivität/ } \\
\text { Beschäftigungsoptionen im } \\
\text { zweiten Arbeitsmarkt }\end{array}$ & $<6$ Monate \\
\hline $\begin{array}{l}\text { 6. Aktivierende Betreuung } \\
\text { (Betreuungskunden) }\end{array}$ & $\begin{array}{l}\text { Die Bearbeitung schwerer } \\
\text { persönlicher/sozialer Probleme }\end{array}$ & $<6$ Monate \\
\hline
\end{tabular}

Quelle: BA-Konzept Handlungsprogramme; eigene Darstellung.

\section{Diskussion und Empfehlungen}

Der Ansatz des Profilings, Dienstleistungen nach Kundenbedarfen zu differenzieren, ist grundsätzlich richtig. Ein zentraler Problembereich der Kundengruppenzuordnung (Profiling) sind aber fehlerhafte Zuordnungen. Insbesondere die falsche Einordnung als Betreuungskunde kann individuell schädlich sein und nachfolgend zu wirtschaftlicher Ineffizienz führen.

Der qualitativen Weiterentwicklung des Profilings kommt eine Schlüsselrolle $\mathrm{zu}$, um eine passgenauere, differenzierte Förderung umsetzen zu können. In der wissenschaftlichen Diskussion wird bezweifelt, ob statistische Profilingsysteme hinreichend genau und vollständig sein können, um ausschließlich darauf die Vermittlungspraxis aufzubauen. Umgekehrt ist vermittlerbasiertes Profiling auch fehleranfällig und bedarf systematischer Kategorien und Vorgehensweisen, um valide Einschätzungen treffen zu können.

Eine Optimierungslösung könnte eine Kombination aus statistischem und vermittlerbasiertem Profiling sein, um die Vorzüge beider Ansätze zu vereinen. Wenn aber das von der Bundesagentur für Arbeit geplante statistische Profilingsystem TREFFER in der Vermittlungspraxis eingeführt werden sollte, dürfte dies nicht sofort das vermittlerbasierte Profiling flächendeckend ersetzen. Vorteilhafter wäre vielmehr, eine experimentelle Einführung und vergleichende Evaluation beider Profilingansätze durch eine externe Einrichtung durchzuführen.

Eine Folge der aktuellen BA-Geschäftspolitik, auf der die Handlungsprogramme aufbauen, bildet der Umstand, dass die „Betreuungskunden“ - Arbeitslose mit den größten Schwierigkeiten am Arbeitsmarkt - zu wenig und zu spät gefördert werden. Viele werden bis zum Ende der Bezugsdauer des Arbeitslosengeldes I "geparkt". Eine gezielte Förderung setzt oft erst mit Bezug des Arbeitslosengeldes II ein. Das bedeutet im Extremfall ein Jahr (eineinhalb Jahre bei Älteren) Lohnersatz, aber keine zielgerichtete Intervention. Diese institutionelle Schwachstelle ist der entscheidende Kritikpunkt, dem sich der Gesetzgeber stellen müsste.

Die institutionell angelegte Gefahr, dass viele Betreuungskunden gar keine bedarfsgerechte Intervention angeboten bekommen, bis ihre SGB-III-Lohnersatzleistungen aufgebraucht sind und sie in den SGB-II-Bereich übergehen, könnte abgeschwächt, wenn nicht gar beseitigt werden. Vier Möglichkeiten böten sich hierfür dem Gesetzgeber generell an:

(1)Abschaffung des Aussteuerungsbetrages: Dies würde zumindest die negativen Anreize für jene SGB-III-Förderungen senken, deren Ende außerhalb des ALG-I-Bezugs liegen. 
(2) Schnittstellen SGB III/II optimieren: Zielpersonen der Kundengruppe 3 (Betreuungskunden) müssten schneller und in Kooperation mit dem SGB-II-Träger gefördert werden, so dass sie nicht ein Jahr ohne Betreuungsleistungen bleiben.

(3) Erweiterung oder Abmilderung Zielsystem: Der Marktersatz müsste durch eine Änderung des SGB III und der BA-Geschäftspolitik und/oder eine Ergänzung durch weitere Sonderprogramme ausgebaut werden.

(4) Massive Ausweitung der Einschaltung Dritter unmittelbar nach Diagnose der Kundengruppe 3:

Mithilfe Dritter könnte versucht werden, die Beschäftigungsfähigkeit dieser Personen zu erhöhen bzw. zu bewahren. Gesetzliche Änderungen an dieser Stelle zögen eine Veränderung der Produkteinsatzlogik der BA nach sich.

Generell bleibt darüber hinaus auf die eingeschränkten Möglichkeiten der Arbeitsvermittlung hinzuweisen. Die Arbeitsvermittlung, in welcher Mischung von öffentlicher und privater Verantwortung auch immer, kann die Lösung der Massenarbeitslosigkeit und die Schließung der vorhandenen Arbeitsplatzlücke nicht leisten. Hier sind vor allem vernünftige Weichenstellungen aus der Wirtschafts-, Finanz- und auch Bildungspolitik gefragt.

\section{Neujustierung der Aktivierung}

Die Qualität und Schnelligkeit der Arbeitsvermittlung sollte im Rahmen der Hartz-Reformen auch mit einer Neuausrichtung der Bewerberaktivierung verbessert werden. Die gesetzlichen Reforminstrumente und der Aktivierungsansatz der BA betonen dabei das „Fordern“ stärker als das „Fördern“. Ausnahme ist die frühzeitige Meldepflicht, die den Arbeitnehmer zur Arbeitsuchendmeldung (nunmehr) drei Monate vor Beendigung eines Arbeits- oder Ausbildungsverhältnisses verpflichtet. Damit soll die Zeitspanne vor Beginn der Arbeitslosigkeit besser genutzt werden, um möglichst eine direkte "Jobzu-Job-Vermittlung“ zu erreichen. Dieses erwies sich in der Praxis als problematisch. Zum einen sind die zu vermittelnden Stellen meist kurzfristig zu besetzen, und zum anderen mangelt es an der Mitarbeit der (noch) beschäftigten Arbeitsuchenden (z.B. Termintreue). Um dieses Instrument zu stärken, erschiene die Einführung verbindlicher Freistellungsregelungen sinnvoll, wie ursprünglich von der Hartz-Kommission empfohlen.

Das von den Reformen anvisierte Ziel eines vereinfachten und flexibler einsetzbaren Sanktionsinstrumentariums ist kaum erreicht worden. Die Quote erhobener und erfolgreicher Widersprüche und Klagen hat sich nach einem Rückgang im ersten Jahr der Umsetzung wieder normalisiert. Gleichzeitig haben Maßnahmen wie verstärkte Nachweise von Eigenbemühungen und erhöhte Kontaktdichte, die als Test der Motivation und Arbeitsbereitschaft für Vermittler im Vordergrund stehen, vor allem die individuellen Lasten erhöht. Demgegenüber wurde die aktive Förderung immer selektiver, kam also weniger Menschen zugute. Der Ansatz der „fordernden Aktivierung" und der Ansatz der "fördernden Aktivierung" sollten daher in eine neue Balance gebracht worden.

\section{Arbeitgeberorientierte Vermittlung}

Durch die seit 2003 laufende Reformwelle in der BA sind merkliche Impulse ausgelöst worden, um die vermittlungsorientierten Dienstleistungen an Arbeitgeber zu verbessern. Wichtige Elemente bilden das Mindestressourcenkontingent für Arbeitgebervermittlung und die Orientierung an festgelegten Qualitätsstandards. Dennoch kann der Bereich noch weiter verstärkt werden. Die Ergebnisse der Evaluation legen nahe, dass Beratungsbedarfe und Stellenentwicklungspotenziale gerade bei den kleinen und Mittelbetrieben bestehen. Hier sollte die Bundesagentur für Arbeit ansetzen.

Für das Dienstleistungsangebot im Bereich Vermittlung und Beratung an Arbeitgeber wäre eine präzisere Konzeptentwicklung sinnvoll, die unterschiedliche Leistungen und Leistungstiefen mit unterschiedlichen Finanzierungsmodi unterlegt. In diesem Kontext kämen eventuell auch Beratungsgutscheine für kleine und mittlere Unternehmen in Betracht.

Das Ausloten des optimalen lokalen Mixes von arbeitgeber- und arbeitnehmerorientierter Vermittlung sollte weiterhin im Ermessen der Agenturen bleiben. Ähnliches gilt für die Zusammenarbeit zwischen arbeitgeber- und arbeitnehmerorientierten Vermittlern, die einer guten Institutionalisierung und fortwährender Pflege in den Agenturen bedarf. Nachdem sich das Geschäftssystem Kundenzentrum (bei notwendiger Mindestgröße) als weithin funktionstüchtig erweist, stellt sich die Frage, ob nach der stark zentralisierten Einführungsphase des Organisationsstandards den Agenturen nicht wieder mehr dezentrale Gestaltungsfreiheiten eingeräumt werden sollten.

\section{Neue Instrumente der Arbeitsvermittlung unter Einschaltung Dritter}

Die vier unter dem Begriff „vermittlungsnahe Dienstleistungen“ zusammengefassten Instrumente bieten ihrer Grundidee nach neue Möglichkeiten öffentlich-privater Zusammenarbeit in der Arbeitsvermittlung (siehe Abb. 3). In der Evaluation wurden sowohl die Eingliederungswirkungen als auch die Implementation dieser Instrumente untersucht. ${ }^{1}$ Die neuen Instrumente haben sich in ihrer bisherigen Form in der Praxis nicht bewährt. Die Implementationsanalyse zeigt zahlreiche Umsetzungsdefizite, verweist aber auch auf exemplarisch gute Praktiken und Erfahrungen vor Ort (vgl. auch Kaps/Schütz 2007).

\section{Der Vermittlungsgutschein $(\S \mathbf{4 2 1 \mathrm { g } )}$}

Die Wirkungsanalyse attestiert allein dem Vermittlungsgutschein eine positive Wirkung. Diese ist allerdings ausschließlich auf die Ergebnisse für ausgegebene 
Gutscheine im Jahre 2005, also nach der Neuordnung des Instruments, zurückzuführen. Gutscheinbesitzer des Jahres 2005 haben im Vergleich zu der Kontrollgruppe häufiger wieder eine Beschäftigung gefunden. Die Beschäftigungsverhältnisse, die durch das Einlösen eines Vermittlungsgutscheins zustande kamen, wurden jedoch vor allem in Ostdeutschland schneller wieder aufgelöst. Dies deutet auf mögliche Mitnahmeeffekte durch die Arbeitgeber hin, deren genaues Ausmaß unklar bleibt. Außerdem zeigt die Analyse der Umsetzung des Instruments Informations- und Qualitätsdefizite auf. Die Ergebnisse der Wirkungsanalyse sollten daher nicht überbewertet werden.

\section{Die Beauftragung Dritter mit der Vermittlung}

Die nach § 37 SGB III mit der Gesamtvermittlung beauftragten privaten Dienstleister haben keine besseren Ergebnisse als die Agenturen erzielen können: Weder die Verweildauer in Arbeitslosigkeit bis zum Übergang in reguläre Beschäftigung noch die Übergangswahrscheinlichkeit unterscheiden sich signifikant von denen der Nichtteilnehmer. Die mit dem § 37 durch private Dienstleister in Arbeit gebrachten Beschäftigten wurden zudem vier Monate früher wieder arbeitslos als die Personen der Kontrollgruppe.

Nur wenn die Beauftragung Dritter mit der Vermittlung nicht als Alternative, sondern als Ergänzung zu den Vermittlungsaktivitäten der Agenturen eingesetzt wird, etwa bei Kapazitätsengpässen, kann die bisherige Tätigkeitsbilanz der Privaten positiver gedeutet werden. Sie vermitteln über Beauftragungen nach $\S 37$ nicht besser, aber auch insgesamt nicht schlechter.

\section{Die Beauftragung von Trägern mit Eingliederungs- maßnahmen}

Als reines Vermittlungsinstrument sind die Eingliederungsmaßnahmen wenig erfolgreich. Im Vergleich mit der Kontrollgruppe wurden weniger Personen in Beschäftigung vermittelt. Im Vergleich zur Vermittlung durch Dritte, zu PSA oder dem Vermittlungsgutschein war die Beschäftigung im Anschluss an eine Eingliederungsmaßnahme jedoch deutlich stabiler. Erfolgreich waren die Eingliederungsmaßnahmen beim Abbau von Vermittlungshemmnissen insbesondere von männlichen Jugendlichen.

Als ein Förderinstrument mit Maßnahmencharakter ermöglichen die Eingliederungsmaßnahmen eine flexible und auf individuelle Bedürfnisse abgestimmte Bündelung von Aktivitäten für spezifische Zielgruppen, die im Instrumentarium des SGB III als solche Kombinationsmöglichkeit sonst nicht enthalten ist. Daher spricht vieles dafür, dieses Instrument beizubehalten, es eher als eine Einstiegsmaßnahme zu gestalten, auf die ggf. weitere Interventionen folgen, bevor eine erfolgreiche Vermittlung stattfinden kann.

\section{Die Personal-Service-Agentur (PSA)}

Nach den Ergebnissen der Wirkungsanalyse waren die PSA in den Jahren 2003/2004 weder effizient noch effektiv. PSA-Beschäftigte hatten eine deutlich geringere Wahrscheinlichkeit, wieder in eine reguläre Beschäftigung eingegliedert zu werden. Die Kontrollgruppe von Nichtteilnehmern war im Durchschnitt einen Monat früher in regulärer Beschäftigung. Allerdings werden Verleihzeiten, in denen PSA-Teilnehmer an Unternehmen ausgeliehen waren, hier nicht berücksichtigt. Der beobachtete Lock-in-Effekt legt nahe, dass der in der PSA angelegte Zielkonflikt zwischen Verleih und Vermittlung der Beschäftigten nicht ausbalanciert ist. Die Vermittlungsprämie müsste so gestaltet sein, dass auch ein gewerbliches Zeitarbeitsunternehmen ausreichend Anreiz hat, einen guten Mitarbeiter zu vermitteln. Zwar ist es positiv zu bewerten, dass mit den neuen Ausschreibungen ab Mitte 2005 die Dauer der Subvention gekürzt wurde. Allerdings hat sich in der Ausschreibung eine stärkere Gewichtung der Vermittlungsprämie nicht durchgesetzt.

\section{Handlungsoptionen zur Weiterentwicklung privater Dienstleistungen in der Arbeitsvermittlung}

Der Gesetzgeber steht vor der Aufgabe, eine grundsätzliche politische Entscheidung über die künftige Aufgabenverteilung zwischen öffentlicher und privater Arbeitsvermittlung zu treffen, die die bisherigen Unschärfen des SGB III und seiner Gesetzesbegründungen beseitigt. Die generellen Handlungsoptionen sind

a) die Abschaffung wirkungsschwacher Vermittlungsinstrumente zur Beauftragung privater Dritter,

b) die Optimierung der vorhandenen Vermittlungsinstrumente und Verfahren,

c) eine grundlegende Neuordnung der Instrumente und Steuerungsmechanismen zur Einbeziehung Dritter in die Vermittlung.

Dazu wäre es auch sinnvoll, die Einkaufsorganisation genauer zu untersuchen, die bisher die Gestaltungsspielräume der Agenturen bei der arbeitsmarktpolitischen Förderung stark einschränkt.

Vor dem Hintergrund der Evaluierungsergebnisse läge die Abschaffung der wirkungsschwachen Instrumente PSA, § 37 und § 421i ziemlich nahe. Ein vollständiger Verzicht auf Förderinstrumente der privaten Arbeitsvermittlung würde allerdings der vom Gesetzgeber ursprünglich intendierten Entwicklung entgegenlaufen, die private bewerberorientierte Vermittlung insgesamt $\mathrm{zu}$ stärken. Zudem wurde die Arbeit der privaten Vermittlungsdienstleister zum Teil durch Prozessprobleme erschwert: Eine stärkere Qualitätsentwicklung der privaten beauftragten Vermittlung wurde durch die Dominanz des Preiswettbewerbs bei der Vergabe über die Regionalen Einkaufszentren behindert (vgl. Gülker/Kaps 2006).

Insbesondere bei den PSA überwiegen aufgrund fehlender Wirkung und hoher Kosten durchaus die Vorteile 


\section{Abbildung 3: Neue Instrumente der Arbeitsvermittlung unter} Einschaltung Dritter

- Der Vermittlungsgutschein nach § 421g SGB III ermöglicht es Arbeitslosen, selbst am freien Markt der Personaldienstleister denjenigen aufzusuchen, der die beste Unterstützung verspricht. Der Gutschein steht allen leistungsberechtigten Arbeitsuchenden nach sechs Wochen Arbeitslosigkeit zu, ein privater Vermittler kann bei erfolgreicher Vermittlung den Gegenwert von aktuell 2000 Euro bei der Agentur für Arbeit einlösen.

- Beauftragung Dritte mit der Vermittlung. Nach $\S 37$ SGB III können private Personalvermittler direkt an den Vermittlungsaufgaben der Arbeitsagenturen beteiligt werden. Die Agenturen können Teilaufträge (z.B. Bewerbungstraining oder Fallmanagement), Stellenakquisition oder auch die gesamte Vermittlung an einen externen Anbieter vergeben. Nach sechs Monaten Arbeitslosigkeit haben Arbeitsuchende einen Rechtsanspruch auf die Betreuung durch einen Vermittler außerhalb der Agentur.

- Im Unterschied dazu handelt es sich bei einer Beauftragung von Trägern mit Eingliederungsmaßnahmen nach $\S 421 \mathrm{i}$ SGB III um eine Gruppenmaßnahme. Besondere Zielgruppen sollen durch einen innovativen Maßnahmezuschnitt in dauerhafte sozialversicherungspflichtige Beschäftigung vermittelt werden. In ihren Methoden sind die Anbieter dabei frei, das beste Konzept soll sich im Wettbewerb durchsetzen.

- Die Personal-Service-Agentur (PSA) ist ein vermittlungsorientiert arbeitendes Zeitarbeitsunternehmen. Arbeitslose werden im Auftrag der Arbeitsagentur für neun Monate sozialversicherungspflichtig eingestellt mit der Erwartung, dass die Zeitarbeitseinsätze eine Brücke in eine dauerhafte Beschäftigung bieten. Die Agentur für Arbeit zahlt der PSA Aufwandsvergütung und Vermittlungsprämie, die PSA wiederum verpflichtet sich, verleihfreie Zeiten für Qualifizierungsmaßnahmen zu nutzen.

einer Abschaffung. Den Arbeitsagenturen sollte aber freigestellt bleiben, ob sie über die Freie Förderung vermittlungsorientierte Arbeitnehmerüberlassung als Integrationsweg nutzen wollen.

Bei einer Beibehaltung des Instruments Beauftragungen Dritter mit Vermittlung (§ 37) sprächen die Ergebnisse allenfalls dafür, diese vor allem zur Behebung von Kapazitätsengpässen und für bestimmte Zielgruppen einzusetzen. Die Eingliederungsmaßnahmen (§ 421i) sind als reines Vermittlungsinstrument ebenfalls wenig erfolgreich. Als Förderinstrument besitzen sie jedoch Potenzial für an individuellen Bedarfen ausgerichtete Zielgruppenmaßnahmen, die in dieser Form im SGB III sonst nicht enthalten sind. Aus dieser Sicht könnte das Instrument beibehalten werden.

Beim Vermittlungsgutschein schließlich sprechen Ergebnisse der Wirkungsanalyse eher für eine Beibehaltung und Weiterentwicklung des Instruments. Vor dem Hintergrund der Umsetzungserfahrungen gälte es allerdings, dessen Informations- und Qualitätsdefizite zu beheben.

Eine grundlegende Alternative bietet der Vorschlag, die bisherigen zentral regulierten Vermittlungsinstrumente abzuschaffen und stattdessen den lokalen Arbeitsagenturen ein Budget zur freien Ausgestaltung ihrer Förderpolitik zu gewähren. Dies wäre ein innovativer Schritt, um die dezentrale Verantwortung der Agenturen zu stärken und so das Leistungspotenzial der Arbeitsmarktpolitik zu verbessern.

\section{Literatur}

Gülker, S. \& P. Kaps 2006. Effizienzsteigerung der Arbeitsvermittlung durch Contracting-Out? Eine Prozessanalyse zur öffentlich-privaten Kooperation bei vermittlungsnahen Dienstleistungen. In: Zeitschrift für Sozialreform, Heft 1/2006, 29-52.

Hess, D., P. Kaps \& H. Mosley 2006. Implementations- und Wirkungsanalyse der Personal-Service-Agentur. In: DIW-Vierteljahrsheft 3/2006, 9-31.

Kaps, P. \& H. Schütz 2007. Privatisierung von Vermittlungsdienstleistungen - Wundermittel für Effizienz? Eine Bestandsaufnahme deutscher und internationaler Erfahrungen. WZB Discussion Paper SP I 2007-101.

Mauer, A. 2007. Wirkungsanalyse der Neuausrichtung der Arbeitsvermittlung im neuen Kundenzentrum. In: Zeitschrfit für Arbeitsmarktforschung (im Erscheinen).

Schütz, H. \& F. Oschmiansky 2006. Arbeitsamt war gestern - Neuausrichtung der Vermittlungsprozesse in der Bundesagentur für Arbeit nach den Hartz-Gesetzen. In: Zeitschrift für Sozialreform, Heft $1 / 2006,5-28$.

WZB/infas 2006 (Autorengemeinschaft). Evaluation der Maßnahmen zur Umsetzung der Vorschläge der Hartz-Kommission. Modul 1a: Neuausrichtung der Vermittlungsprozesse. Endbericht 2006. Berlin, Bonn. (http://www.wzb.eu/ars/ab/)

\section{Fußnote}

1 Außerdem wurde eine ergänzende Befragung von insgesamt rund 5.000 Teilnehmern und Nichtteilnehmern durchgeführt. 